Manuscript: jp0642995

\title{
A task-specific ionic liquid for solubilizing metal oxides
}

Peter Nockemann, Ben Thijs, Stijn Pittois, Jan Thoen, Christ Glorieux, Kristof Van Hecke, Luc Van Meervelt, Barbara Kirchner, Koen Binnemans*

Supporting Information 
Figure S1. Packing of the molecules in the crystal structure of protonated betaine bis(trifluoromethylsulfonyl)imide [Hbet][Tf $2 \mathrm{~N}]$.

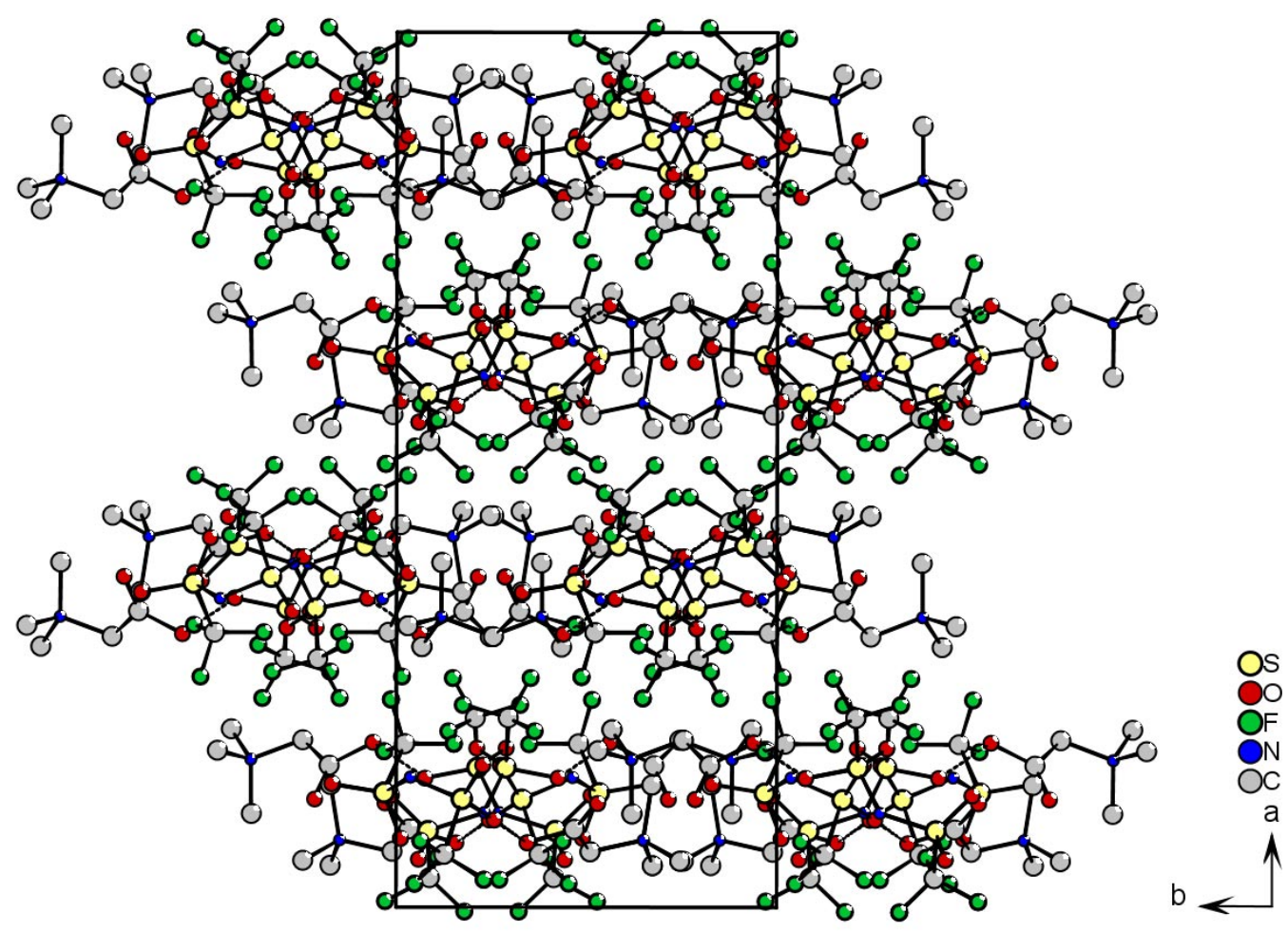


Figure S2. Packing of the molecules in the crystal structure of protonated betaine bis(trifluoromethylsulfonyl)imide $\left[(\text { Hbet })_{3}(\right.$ bet $\left.)\right]\left[\mathrm{Tf}_{2} \mathrm{~N}\right]_{3}$

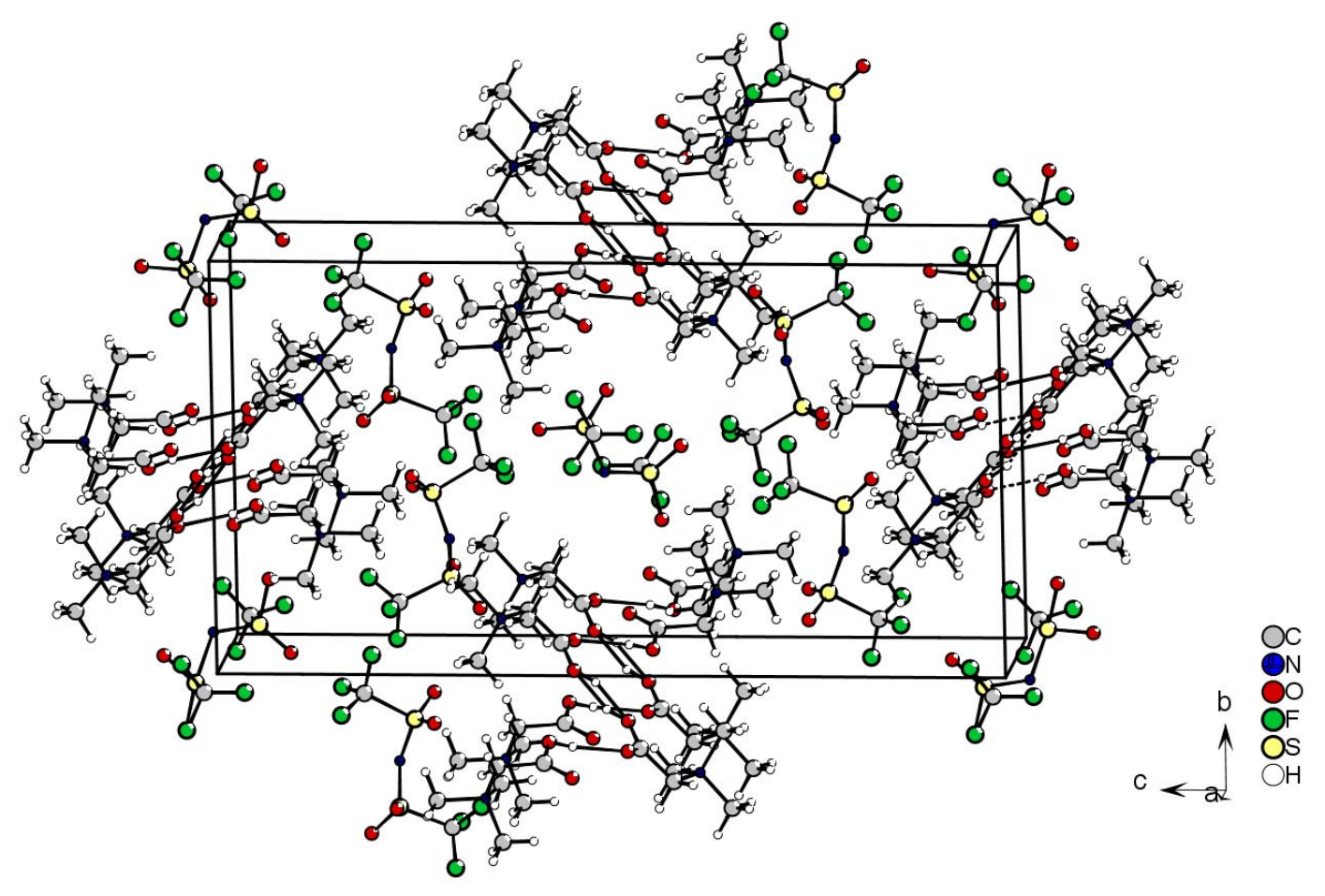


Figure S3: Crystal structure of $\left[\mathrm{Cu}(\text { bet })_{4}\left(\mathrm{H}_{2} \mathrm{O}\right)_{2}\right]\left[\mathrm{Cu}_{2}(\text { bet })_{4}\left(\mathrm{H}_{2} \mathrm{O}\right)_{2}\right]\left[\mathrm{Tf}_{2} \mathrm{~N}\right]_{6}$

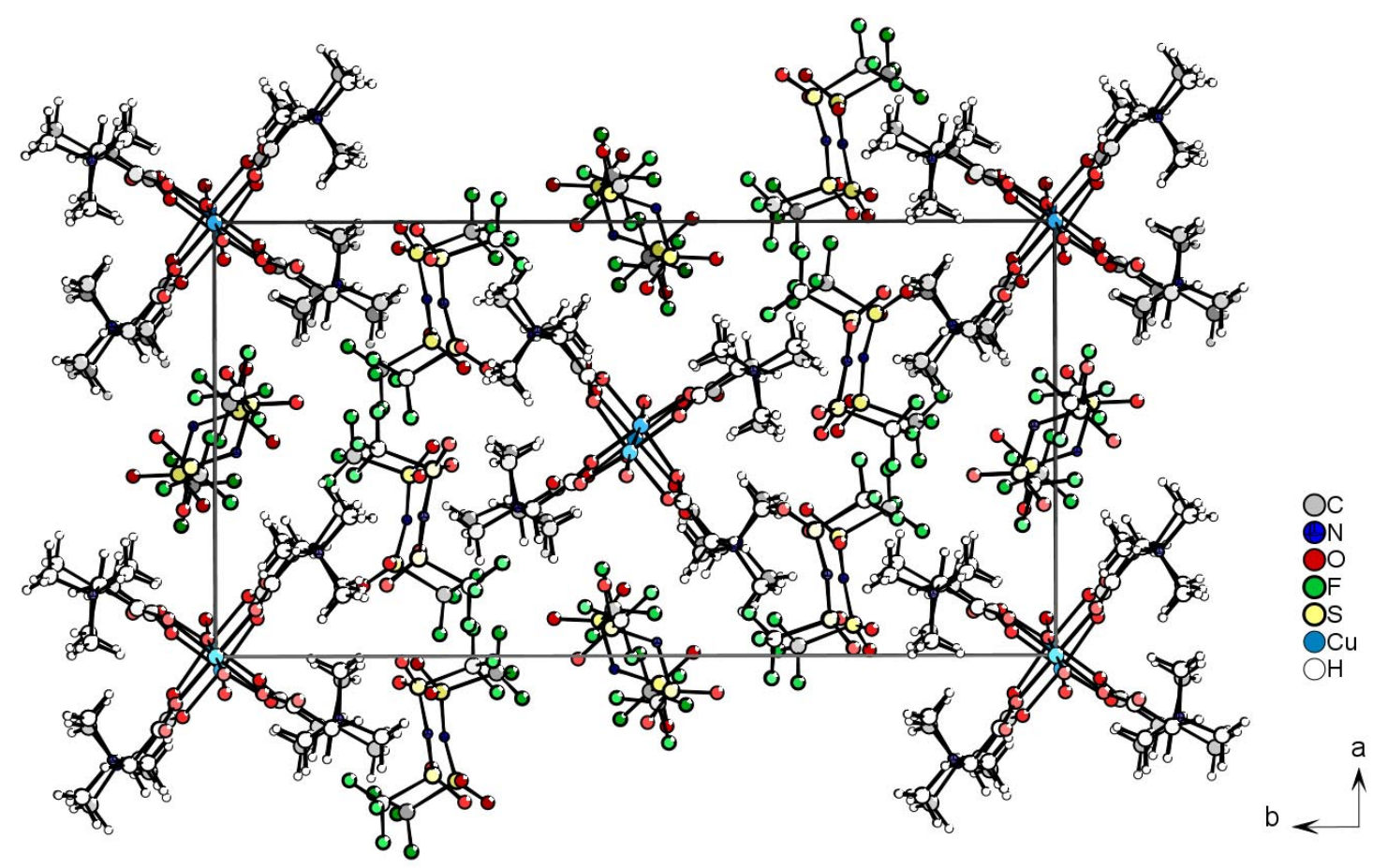


Figure S4. Crystal structure of $\left[\mathrm{Dy}{ }_{2}(\mathrm{bet})_{8}\left(\mathrm{H}_{2} \mathrm{O}\right)_{4}\right]\left[\mathrm{Tf}_{2} \mathrm{~N}\right]_{6}$

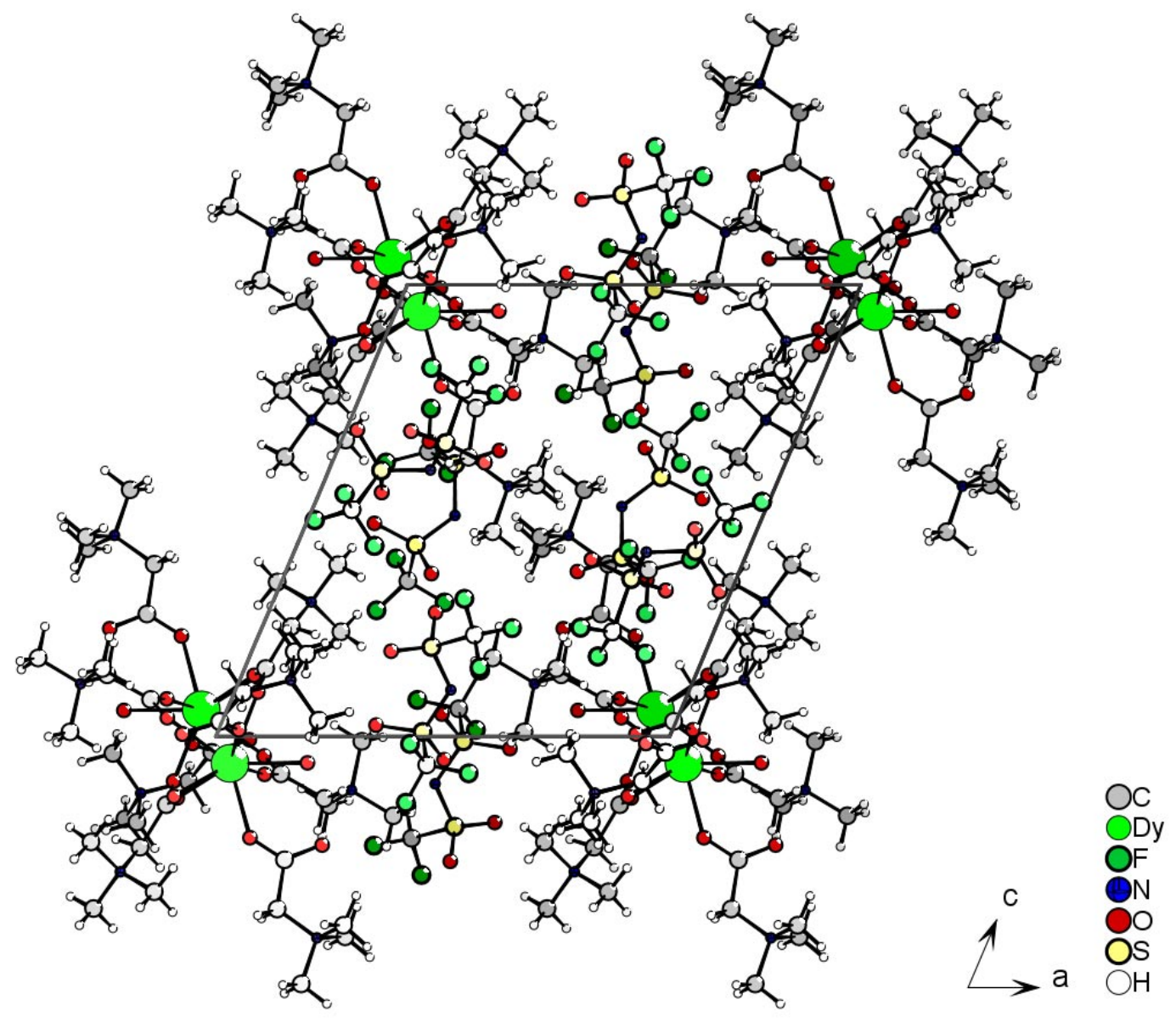




\section{Synthesis and characterization of metal complexes}

\section{$\underline{\text { Yttrium(III) complex }}$}

$\mathrm{Y}_{2} \mathrm{O}_{3}(1 \mathrm{~g} ; 4.42 \mathrm{mmol})$ was mixed with $\left[\right.$ Hbet] $\left[\mathrm{Tf}_{2} \mathrm{~N}\right](10.583 \mathrm{~g} ; 26.5 \mathrm{mmol})$ and $10 \mathrm{~mL}$ of water. The mixture was stirred under reflux for 12 hours. After filtration, water was evaporated under vacuum. The white crystals were recrystallized from water 3 times. Elemental analysis: calcd(\%) for $\mathrm{Y}_{2}\left(\left(\mathrm{CH}_{3}\right)_{3} \mathrm{NCH}_{2} \mathrm{CO}_{2}\right)_{8}\left(\mathrm{~N}\left(\mathrm{SO}_{2} \mathrm{CF}_{3}\right)_{2}\right)_{6}\left(\mathrm{H}_{2} \mathrm{O}\right)_{2}\left(\mathrm{M}_{\mathrm{w}}=\right.$ $2831.90 \mathrm{~g} \mathrm{~mol}^{-1}$ ) C 22.05, H 3.27, N 6.92; found C 21.67, H 3.03, N 6.51.

\section{$\underline{\text { Lanthanum(III) complex }}$}

$\mathrm{La}_{2} \mathrm{O}_{3}(1 \mathrm{~g} ; 3.06 \mathrm{mmol})$ was mixed with $\left[\right.$ Hbet] $\left[\mathrm{Tf}_{2} \mathrm{~N}\right](7.334 \mathrm{~g} ; 18.4 \mathrm{mmol})$ and $10 \mathrm{~mL}$ of water. The mixture was stirred under reflux for 12 hours. After filtration, water was evaporated under vacuum. The white crystals were recrystallized from water 3 times. Elemental analysis: calcd(\%) for $\mathrm{La}_{2}\left(\left(\mathrm{CH}_{3}\right)_{3} \mathrm{NCH}_{2} \mathrm{CO}_{2}\right)_{8}\left(\mathrm{~N}\left(\mathrm{SO}_{2} \mathrm{CF}_{3}\right)_{2}\right)_{6}\left(\mathrm{H}_{2} \mathrm{O}\right)_{4}\left(\mathrm{M}_{\mathrm{w}}=\right.$ $2967.93 \mathrm{~g} \mathrm{~mol}^{-1}$ ) C 21.04, H 3.24, N 6.60; found C 20.58, H 3.24, N 6.19.

\section{Praseodymium(III) complex}

$\operatorname{Pr}_{6} \mathrm{O}_{11}(1 \mathrm{~g} ; 0.97 \mathrm{mmol})$ was mixed with $[$ Hbet $]\left[\mathrm{Tf}_{2} \mathrm{~N}\right](7.018 \mathrm{~g} ; 17.6 \mathrm{mmol})$ and $10 \mathrm{~mL}$ of water. The mixture was stirred under reflux for 12 hours. After filtration, water was evaporated under vacuum. The white crystals were recrystallized from water 3 times. Elemental analysis: calcd(\%) for $\operatorname{Pr}_{2}\left(\left(\mathrm{CH}_{3}\right)_{3} \mathrm{NCH}_{2} \mathrm{CO}_{2}\right)_{8}\left(\mathrm{~N}\left(\mathrm{SO}_{2} \mathrm{CF}_{3}\right)_{2}\right)_{6}\left(\mathrm{H}_{2} \mathrm{O}\right)_{4}(2971.94 \mathrm{~g}$ $\mathrm{mol}^{-1}$ ) C 21.02, H 3.26, N 6.53; found C 20.61, H 4.08, N 6.16. 


\section{$\underline{\text { Neodymium(III) complex }}$}

$\mathrm{Nd}_{2} \mathrm{O}_{3}(1 \mathrm{~g} ; 2.97 \mathrm{mmol})$ was mixed with $[\mathrm{Hbet}]\left[\mathrm{Tf}_{2} \mathrm{~N}\right](7.102 \mathrm{~g} ; 17.8 \mathrm{mmol})$ and $10 \mathrm{~mL}$ of water. The mixture was stirred under reflux for 12 hours. After filtration, water was evaporated under vacuum. The purple crystals were recrystallized from water 3 times. Elemental analysis: calcd(\%) for $\mathrm{Nd}_{2}\left(\left(\mathrm{CH}_{3}\right)_{3} \mathrm{NCH}_{2} \mathrm{CO}_{2}\right)_{8}\left(\mathrm{~N}\left(\mathrm{SO}_{2} \mathrm{CF}_{3}\right)_{2}\right)_{6}\left(\mathrm{H}_{2} \mathrm{O}\right)_{4}\left(\mathrm{M}_{\mathrm{w}}=\right.$ $2978.60 \mathrm{~g} \mathrm{~mol}^{-1}$ ) C 20.96, H 3.24, N 6.58; found C 20.60, H 3.22, N 6.20.

\section{$\underline{\text { Samarium(III) complex }}$}

$\mathrm{Sm}_{2} \mathrm{O}_{3}(1 \mathrm{~g} ; 2.86 \mathrm{mmol})$ was mixed with $[\mathrm{Hbet}]\left[\mathrm{Tf}_{2} \mathrm{~N}\right](6.852 \mathrm{~g} ; 17.20 \mathrm{mmol})$ and $10 \mathrm{~mL}$ water. The mixture was stirred under reflux for 12 hours. After filtration, water was evaporated under vacuum. The white crystals were recrystallized from water 3 times. Elemental analysis: $\operatorname{calcd}(\%)$ for $\mathrm{Sm}_{2}\left(\left(\mathrm{CH}_{3}\right)_{3} \mathrm{NCH}_{2} \mathrm{CO}_{2}\right)_{8}\left(\mathrm{~N}\left(\mathrm{SO}_{2} \mathrm{CF}_{3}\right)_{2}\right)_{6}\left(\mathrm{H}_{2} \mathrm{O}\right)_{2}\left(\mathrm{M}_{\mathrm{w}}=\right.$ $2954.81 \mathrm{~g} \mathrm{~mol}^{-1}$ ) C 21.13, H 3.13, N 6.60; found C 21.10, H 3.10, N 6.05.

\section{Europium(III) complex}

$\mathrm{Eu}_{2} \mathrm{O}_{3}(1 \mathrm{~g} ; 2.84 \mathrm{mmol})$ was mixed with $[\mathrm{Hbet}]\left[\mathrm{Tf}_{2} \mathrm{~N}\right](6.789 \mathrm{~g} ; 17.04 \mathrm{mmol})$ and $10 \mathrm{~mL}$ of water. The mixture was stirred under reflux for 12 hours. After filtration, water was evaporated under vacuum. The white crystals were recrystallized from water 3 times. Elemental analysis: calcd $(\%)$ for $\mathrm{Eu}_{2}\left(\left(\mathrm{CH}_{3}\right)_{3} \mathrm{NCH}_{2} \mathrm{CO}_{2}\right)_{8}\left(\mathrm{~N}\left(\mathrm{SO}_{2} \mathrm{CF}_{3}\right)_{2}\right)_{6}\left(\mathrm{H}_{2} \mathrm{O}\right)_{2}\left(\mathrm{M}_{\mathrm{w}}=\right.$ $2958.01 \mathrm{~g} \mathrm{~mol}^{-1}$ ) C 21.11, H 3.13, N 6.62; found C 20.25, H 3.08, N 6.32. 


\section{Gadolinium(III) complex}

$\mathrm{Gd}_{2} \mathrm{O}_{3}(1 \mathrm{~g} ; 2.75 \mathrm{mmol})$ was mixed with [Hbet][Tf $\left.{ }_{2} \mathrm{~N}\right](6.592 \mathrm{~g} ; 16.5 \mathrm{mmol})$ and $10 \mathrm{~mL}$ of water. The mixture was stirred under reflux for 12 hours. After filtration, water was evaporated under vacuum. The white crystals were recrystallized from water 3 times. Elemental analysis: $\operatorname{calcd}(\%)$ for $\mathrm{Gd}_{2}\left(\left(\mathrm{CH}_{3}\right)_{3} \mathrm{NCH}_{2} \mathrm{CO}_{2}\right)_{8}\left(\mathrm{~N}\left(\mathrm{SO}_{2} \mathrm{CF}_{3}\right)_{2}\right)_{6}\left(\mathrm{H}_{2} \mathrm{O}\right)_{2}\left(\mathrm{M}_{\mathrm{w}}=\right.$ $2968.00 \mathrm{~g} \mathrm{~mol}^{-1}$ ) C 21.04, H 3.10, N 6.60; found C 20.31, H 3.03, N 6.21.

\section{Terbium(III) complex}

$\mathrm{Tb}_{4} \mathrm{O}_{7}(1 \mathrm{~g} ; 1.33 \mathrm{mmol})$ was mixed with $\left[\right.$ Hbet] $\left[\mathrm{Tf}_{2} \mathrm{~N}\right](6.398 \mathrm{~g} ; 16.05 \mathrm{mmol})$ and $10 \mathrm{~mL}$ of water. The mixture was stirred under reflux for 12 hours. After filtration, water was evaporated under vacuum. The white crystals were recrystallized from water 3 times. Elemental analysis: calcd $(\%)$ for $\mathrm{Tb}_{2}\left(\left(\mathrm{CH}_{3}\right)_{3} \mathrm{NCH}_{2} \mathrm{CO}_{2}\right)_{8}\left(\mathrm{~N}\left(\mathrm{SO}_{2} \mathrm{CF}_{3}\right)_{2}\right)_{6}\left(\mathrm{H}_{2} \mathrm{O}\right)_{2}\left(\mathrm{M}_{\mathrm{w}}=\right.$ $2971.94 \mathrm{~g} \mathrm{~mol}^{-1}$ ) C 21.01, H 3.12, N 6.60; found C 20.70, H 3.17, N 6.18.

\section{Dysprosium(III) complex}

$\mathrm{Dy}_{2} \mathrm{O}_{3}(1 \mathrm{~g} ; 2.68 \mathrm{mmol})$ was mixed with $[\mathrm{Hbet}]\left[\mathrm{Tf}_{2} \mathrm{~N}\right](6.402 \mathrm{~g} ; 16.08 \mathrm{mmol})$ and $10 \mathrm{~mL}$ of water. The mixture was stirred under reflux for 12 hours. After filtration, water was evaporated under vacuum. The white crystals were recrystallized from water 3 times. Elemental analysis: $\operatorname{calcd}(\%)$ for $\mathrm{Dy}_{2}\left(\left(\mathrm{CH}_{3}\right)_{3} \mathrm{NCH}_{2} \mathrm{CO}_{2}\right)_{8}\left(\mathrm{~N}\left(\mathrm{SO}_{2} \mathrm{CF}_{3}\right)_{2}\right)_{6}\left(\mathrm{H}_{2} \mathrm{O}\right)_{2}\left(\mathrm{M}_{\mathrm{w}}=\right.$

$2979.09 \mathrm{~g} \mathrm{~mol}^{-1}$ ) C 20.93, H 3.11, N 6.57; found C 20.74, H 3.69, N 6.20. 


\section{$\underline{\text { Holmium(III) complex }}$}

$\mathrm{Ho}_{2} \mathrm{O}_{3}(1 \mathrm{~g} ; 2.64 \mathrm{mmol})$ was mixed with $[\mathrm{Hbet}]\left[\mathrm{Tf}_{2} \mathrm{~N}\right](6.319 \mathrm{~g} ; 15.8 \mathrm{mmol})$ and $10 \mathrm{~mL}$ of water. The mixture was stirred under reflux for 12 hours. After filtration, water was evaporated under vacuum. The purple crystals were recrystallized from water 3 times.

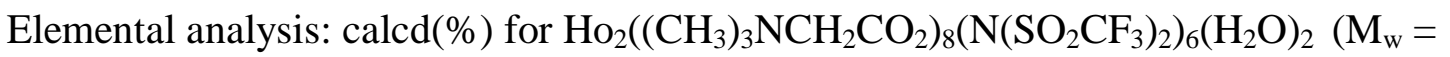
$2983.95 \mathrm{~g} \mathrm{~mol}^{-1}$ ) C 20.92, H 3.11, N 6.57; found C 20.30, H 3.47, N 6.33.

\section{$\underline{\text { Erbium(III) complex }}$}

$\mathrm{Er}_{2} \mathrm{O}_{3}(1 \mathrm{~g} ; 2.61 \mathrm{mmol})$ was mixed with $[\mathrm{Hbet}]\left[\mathrm{Tf}_{2} \mathrm{~N}\right](6.247 \mathrm{~g} ; 15.6 \mathrm{mmol})$ and $10 \mathrm{~mL}$ of water. The mixture was stirred under reflux for 12 hours. After filtration, water was evaporated under vacuum. The white crystals were recrystallized from water 3 times. Elemental analysis: calcd(\%) for $\mathrm{Er}_{2}\left(\left(\mathrm{CH}_{3}\right)_{3} \mathrm{NCH}_{2} \mathrm{CO}_{2}\right)_{8}\left(\mathrm{~N}\left(\mathrm{SO}_{2} \mathrm{CF}_{3}\right)_{2}\right)_{6}\left(\mathrm{H}_{2} \mathrm{O}\right)_{2}\left(\mathrm{M}_{\mathrm{w}}=\right.$ $2988.61 \mathrm{~g} \mathrm{~mol}^{-1}$ ) C 20.90, H 3.10, N 6.56; found C 20.67, H 3.01, N 6.16.

\section{Thulium(III) complex}

$\mathrm{Tm}_{2} \mathrm{O}_{3}(1 \mathrm{~g} ; 2.59 \mathrm{mmol})$ was mixed with $[$ Hbet][Tf $2 \mathrm{~N}](6.193 \mathrm{~g} ; 15.5 \mathrm{mmol})$ and $10 \mathrm{~mL}$ of water. The mixture was stirred under reflux for 12 hours. After filtration, water was evaporated under vacuum. The white crystals were recrystallized from water 3 times. Elemental analysis: $\operatorname{calcd}(\%)$ for $\mathrm{Tm}_{2}\left(\left(\mathrm{CH}_{3}\right)_{3} \mathrm{NCH}_{2} \mathrm{CO}_{2}\right)_{8}\left(\mathrm{~N}\left(\mathrm{SO}_{2} \mathrm{CF}_{3}\right)_{2}\right)_{6}\left(\mathrm{H}_{2} \mathrm{O}\right)_{2}\left(\mathrm{M}_{\mathrm{w}}=\right.$ $2991.96 \mathrm{~g} \mathrm{~mol}^{-1}$ ) C 20.86, H 3.10, N 6.55; found C 20.54, H 3.26, N 6.13. 


\section{$\underline{\text { Ytterbium(III) complex }}$}

$\mathrm{Yb}_{2} \mathrm{O}_{3}(1 \mathrm{~g} ; 2.53 \mathrm{mmol})$ was mixed with $[\mathrm{Hbet}]\left[\mathrm{Tf}_{2} \mathrm{~N}\right](6.064 \mathrm{~g} ; 15.2 \mathrm{mmol})$ and $10 \mathrm{~mL}$ of water. The mixture was stirred under reflux for 12 hours. After filtration, water was evaporated under vacuum. The white crystals were recrystallized from water 3 times. Elemental analysis: $\operatorname{calcd}(\%)$ for $\mathrm{Yb}_{2}\left(\left(\mathrm{CH}_{3}\right)_{3} \mathrm{NCH}_{2} \mathrm{CO}_{2}\right)_{8}\left(\mathrm{~N}\left(\mathrm{SO}_{2} \mathrm{CF}_{3}\right)_{2}\right)_{6}\left(\mathrm{H}_{2} \mathrm{O}\right)_{2}\left(\mathrm{M}_{\mathrm{w}}=\right.$ $3000.17 \mathrm{~g} \mathrm{~mol}^{-1}$ ) C 20.81, H 3.09, N 6.53; found C 20.60, H 3.22, N 6.20.

\section{$\underline{\text { Lutetium(III) complex }}$}

$\mathrm{Lu}_{2} \mathrm{O}_{3}(1 \mathrm{~g} ; 2.51 \mathrm{mmol})$ was mixed with $[\mathrm{Hbet}]\left[\mathrm{Tf}_{2} \mathrm{~N}\right](6.005 \mathrm{~g} ; 15.7 \mathrm{mmol})$ and $10 \mathrm{~mL}$ of water. The mixture was stirred under reflux for 12 hours. After filtration, water was evaporated under vacuum. The white crystals were recrystallized from water 3 times. Elemental analysis: calcd(\%) for $\mathrm{Lu}_{2}\left(\left(\mathrm{CH}_{3}\right)_{3} \mathrm{NCH}_{2} \mathrm{CO}_{2}\right)_{8}\left(\mathrm{~N}\left(\mathrm{SO}_{2} \mathrm{CF}_{3}\right)_{2}\right)_{6}\left(\mathrm{H}_{2} \mathrm{O}\right)_{2}\left(\mathrm{M}_{\mathrm{w}}=\right.$ $3004.02 \mathrm{~g} \mathrm{~mol}^{-1}$ ) C 20.78, H 3.08, N 6.53; found C 20.61, H 4.08, N 6.16.

\section{Copper(II) complex}

$\mathrm{CuO}(1 \mathrm{~g} ; 12.57 \mathrm{mmol})$ was mixed with $\left[\right.$ Hbet] $\left[\mathrm{Tf}_{2} \mathrm{~N}\right](10.014 \mathrm{~g} ; 25.1 \mathrm{mmol})$ and $10 \mathrm{~mL}$ of water. The mixture was stirred under reflux for 12 hours. After filtration, water was evaporated under vacuum. The green crystals were recrystallized from water 3 times. Elemental analysis: $\operatorname{calcd}(\%)$ for $\left.\mathrm{Cu}\left(\left(\mathrm{CH}_{3}\right)_{3} \mathrm{CO}_{2}\right) \mathrm{N}\left(\mathrm{SO}_{2} \mathrm{CF}_{3}\right)_{2}\right)_{2}\left(\mathrm{Mw}=858.14 \mathrm{~g} \mathrm{~mol}^{-1}\right)$. C 19.59, H 2.58, N 6.52; found C 19.55, H 2.94, N 6.30.

Reaction: $\left.\mathrm{CuO}+2\left(\mathrm{CH}_{3}\right)_{3} \mathrm{NCH}_{2} \mathrm{CO}_{2}\right) \mathrm{NH}\left(\mathrm{SO}_{2} \mathrm{CF}_{3}\right)_{2} \rightarrow$ $\mathrm{Cu}\left(\left(\mathrm{CH}_{3}\right)_{3} \mathrm{NCH}_{2} \mathrm{CO} 2\right)\left(\mathrm{N}\left(\mathrm{SO}_{2} \mathrm{CF}_{3}\right)_{2}\right)_{2}+\mathrm{H}_{2} \mathrm{O}$ 


\section{$\underline{\text { Zinc(II) complex }}$}

$\mathrm{ZnO}(1 \mathrm{~g} ; 12.3 \mathrm{mmol})$ was mixed with $[\mathrm{Hbet}]\left[\mathrm{Tf}_{2} \mathrm{~N}\right](9.788 \mathrm{~g} ; 24.5 \mathrm{mmol})$ and $10 \mathrm{~mL}$ of water. The mixture was stirred under reflux for 12 hours. After filtration, water was evaporated under vacuum. The white crystals were recrystallized from water 3 times. Elemental analysis: calcd(\%) for $\mathrm{Zn}_{4}\left(\left(\mathrm{CH}_{3}\right)_{3} \mathrm{NCH}_{2} \mathrm{CO}_{2}\right)_{10}\left(\mathrm{~N}\left(\mathrm{SO}_{2} \mathrm{CF}_{3}\right)_{2}\right)_{8}\left(\mathrm{H}_{2} \mathrm{O}\right)_{4}\left(\mathrm{M}_{\mathrm{w}}=\right.$ $3746.27 \mathrm{~g} \mathrm{~mol}^{-1}$ ) C 21.15 H 3.17, N 6.72; found C 20.47, H 3.48 N 6.42

\section{$\underline{\operatorname{Mercury}(\text { II }) \text { complex }}$}

$\mathrm{HgO}(1 \mathrm{~g} ; 4.6 \mathrm{mmol})$ was mixed with $[\mathrm{Hbet}]\left[\mathrm{Tf}_{2} \mathrm{~N}\right](3.679 \mathrm{~g} ; 9.23 \mathrm{mmol})$ and $10 \mathrm{~mL}$ of water. The mixture was stirred under reflux for 12 hours. After filtration, water was evaporated under vacuum. The white crystals were recrystallized from water 3 times.

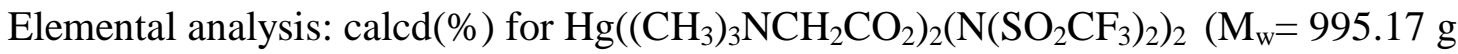
$\mathrm{mol}^{-1}$ ) C 16.89, H 2.23, N 5.62; found C 17.11, H 2.28, N 5.48

\section{$\underline{\text { Manganese(II) complex }}$}

$\mathrm{MnO}(1 \mathrm{~g} ; 14.09 \mathrm{mmol})$ was mixed with $[$ Hbet $]\left[\mathrm{Tf}_{2} \mathrm{~N}\right](11.2 \mathrm{~g} ; 28.1 \mathrm{mmol})$ and $10 \mathrm{~mL}$ of water. The mixture was stirred under reflux for 12 hours. After filtration, water was evaporated under vacuum. The purple crystals were recrystallized from water 3 times. Elemental analysis: calcd(\%) for $\mathrm{Mn}\left(\left(\mathrm{CH}_{3}\right)_{3} \mathrm{NCH}_{2} \mathrm{CO}_{2}\right)_{2}\left(\mathrm{~N}\left(\mathrm{SO}_{2} \mathrm{CF}_{3}\right)_{2}\right)_{2}\left(\mathrm{H}_{2} \mathrm{O}\right)\left(\mathrm{M}_{\mathrm{w}}=\right.$ $867.54 \mathrm{~g} \mathrm{~mol}^{-1}$ ) C $19.38 \mathrm{H} \mathrm{2.78,} \mathrm{N} \mathrm{6.45;} \mathrm{found} \mathrm{C} \mathrm{19.49,} \mathrm{H} \mathrm{2.83,} \mathrm{N} \mathrm{6.28}$ 


\section{$\underline{\text { Silver(I) complex }}$}

$\mathrm{Ag}_{2} \mathrm{O}(1 \mathrm{~g} ; 4.35 \mathrm{mmol})$ was mixed with $[$ Hbet $]\left[\mathrm{Tf}_{2} \mathrm{~N}\right](1.733 \mathrm{~g} ; 4.35 \mathrm{mmol})$ and $10 \mathrm{~mL}$ of water. The mixture was stirred at room temperature and protected from light for 12 hours. After filtration, water was evaporated under vacuum. The white crystals were recrystallized 3 times from water.

Elemental analysis: calcd(\%) for $\mathrm{Ag}_{2}\left(\left(\mathrm{CH}_{3}\right)_{3} \mathrm{NCH}_{2} \mathrm{CO}_{2}\right)_{2}\left(\mathrm{~N}\left(\mathrm{SO}_{2} \mathrm{CF}_{3}\right)_{2}\right)_{2}\left(\mathrm{M}_{\mathrm{w}}=505,16 \mathrm{~g}\right.$ $\mathrm{mol}^{-1}$ ) C 16.64 H 2.19, N 5.54; found C 16.79, H 2.27, N 5.31

\section{$\underline{\text { Nickel(II) complex }}$}

$\mathrm{NiO}(1 \mathrm{~g} ; 13.38 \mathrm{mmol})$ was mixed with [Hbet][Tf $\left.{ }_{2} \mathrm{~N}\right](10.66 \mathrm{~g} ; 26.7 \mathrm{mmol})$ and $10 \mathrm{~mL}$ of water. The mixture was stirred under reflux for 12 hours. After filtration, water was evaporated under vacuum. The green crystals were recrystallized from water 3 times. Elemental analysis: calcd(\%) for $\mathrm{Ni} 2\left(\left(\mathrm{CH}_{3}\right)_{3} \mathrm{NCH}_{2} \mathrm{CO}_{2}\right)_{2}\left(\mathrm{~N}\left(\mathrm{SO}_{2} \mathrm{CF}_{3}\right)_{2}\right)_{2}\left(\mathrm{M}_{\mathrm{w}}=1895.77 \mathrm{~g}\right.$ $\mathrm{mol}^{-1}$ ). C 20.90, H 3.35, N 6.65; found C 20.93, H 3.23, N $6.46 \%$.

\section{Palladium(II) complex}

$\mathrm{PdO}(1 \mathrm{~g} ; 8.16 \mathrm{mmol})$ was mixed with $[\mathrm{Hbet}]\left[\mathrm{Tf}_{2} \mathrm{~N}\right](6.508 \mathrm{~g} ; 16.3 \mathrm{mmol})$ and $10 \mathrm{~mL}$ of water. The mixture was stirred under reflux for 12 hours. After filtration, water was evaporated under vacuum. The yellow crystals were recrystallized from water 3 times. Elemental analysis: calcd $(\%)$ for $\mathrm{Pd}\left(\left(\mathrm{CH}_{3}\right)_{3} \mathrm{NCH}_{2} \mathrm{CO}_{2}\right)_{3}\left(\mathrm{~N}\left(\mathrm{SO}_{2} \mathrm{CF}_{3}\right)_{2}\right)_{2}\left(\mathrm{H}_{2} \mathrm{O}\right)_{2}\left(\mathrm{M}_{\mathrm{w}}=\right.$

$1054.19 \mathrm{~g} \mathrm{~mol}^{-1}$ ) C 21.65, H 3.53, N 6.64; found C 21.31, H 3.02, N 6.80 


\section{$\underline{\text { Lithium salt }}$}

[Hbet][Tf $\left.{ }_{2} \mathrm{~N}\right](16.63 \mathrm{~g} ; 41.75 \mathrm{mmol})$ was added to $10 \mathrm{~mL}$ of an aqueous solution of $\mathrm{LiOH}$ ( $1 \mathrm{~g} ; 41.75 \mathrm{mmol}$ ) and stirred for 15 minutes. After filtration, the water was evaporated under vacuum. The colorless crystals were recrystallized from water and dried at $50^{\circ} \mathrm{C}$ in a vacuum oven. Elemental analysis: calcd $(\%)$ for $\mathrm{Li}\left(\left(\mathrm{CH}_{3}\right)_{3} \mathrm{NCH}_{2} \mathrm{CO}_{2}\right)\left(\mathrm{N}_{(}\left(\mathrm{SO}_{2} \mathrm{CF}_{3}\right)_{2}\right)$ $\left(\mathrm{M}_{\mathrm{w}}=404.23 \mathrm{~g} \mathrm{~mol}^{-1}\right) \mathrm{C} 20.80, \mathrm{H} 2.74, \mathrm{~N} 6.92$; found C 20.69, H 3.07, N 6.76

\section{$\underline{\text { Sodium salt }}$}

[Hbet][Tf $\left.\mathrm{T}_{2} \mathrm{~N}\right](9.96 \mathrm{~g} ; 25.0 \mathrm{mmol})$ was added to $10 \mathrm{~mL}$ of an aqueous solution of $\mathrm{NaOH}$ $(1 \mathrm{~g} ; 25.0 \mathrm{mmol})$ and stirred for 15 minutes. After filtration, the water was evaporated under vacuum. The colorless crystals were recrystallized from water and dried at $50^{\circ} \mathrm{C}$ in a vacuum oven. Elemental analysis: calcd (\%) for $\mathrm{Na}\left(\left(\mathrm{CH}_{3}\right)_{3} \mathrm{NCH}_{2} \mathrm{CO}_{2}\right)\left(\mathrm{N}\left(\mathrm{SO}_{2} \mathrm{CF}_{3}\right)_{2}\right)\left(\mathrm{H}_{2} \mathrm{O}\right)\left(\mathrm{M}_{\mathrm{w}}=438.2 \mathrm{~g} \mathrm{~mol}^{-1}\right) \mathrm{C}$ 19.18, H 2.98, N 6.39; found C 19.78, H 2.97, N 6.38

\section{Potassium salt}

[Hbet][Tf $2 \mathrm{~N}](7.10 \mathrm{~g} ; 17.82 \mathrm{mmol})$ was added to $10 \mathrm{~mL}$ of an aqueous solution of $\mathrm{KOH}$ ( $1 \mathrm{~g} ; 17.82 \mathrm{mmol})$ and stirred for 15 minutes. After filtration, the water was evaporated under vacuum. The colorless crystals were recrystallized from water and dried at $50^{\circ} \mathrm{C}$ in a vacuum oven. Elemental analysis: calcd (\%) for $\mathrm{K}\left(\left(\mathrm{CH}_{3}\right)_{3} \mathrm{NCH}_{2} \mathrm{CO}_{2}\right)\left(\mathrm{N}\left(\mathrm{SO}_{2} \mathrm{CF}_{3}\right)_{2}\right)\left(\mathrm{H}_{2} \mathrm{O}\right)\left(\mathrm{M}_{\mathrm{w}}=454.41 \mathrm{~g} \mathrm{~mol}^{-1}\right) \mathrm{C}$ 18.50, H 2.88, N 6.16; found C 18.83, H 2.82, N 6.18 


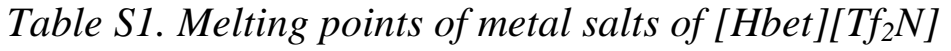

\begin{tabular}{ll}
\hline Compound & Melting point $\left({ }^{\circ} \mathrm{C}\right)$ \\
\hline$[\mathrm{Li}($ bet $)]\left[\mathrm{Tf}_{2} \mathrm{~N}\right]$ & 127 \\
{$[\mathrm{Na}($ bet $)]\left[\mathrm{Tf}_{2} \mathrm{~N}\right] \cdot \mathrm{H}_{2} \mathrm{O}$} & 109 \\
{$[\mathrm{~K}($ bet $)]\left[\mathrm{Tf}_{2} \mathrm{~N}\right] \cdot \mathrm{H}_{2} \mathrm{O}$} & 152 \\
{$\left[\mathrm{Cu}_{3}(\text { bet })_{8}\left(\mathrm{H}_{2} \mathrm{O}\right)_{4}\right]\left[\mathrm{Tf}_{2} \mathrm{~N}\right]_{6}$} & 288 \\
{$\left[\mathrm{Zn}_{4}(\text { bet })_{10}\right]\left[\mathrm{Tf}_{2} \mathrm{~N}\right]_{8}\left(\mathrm{H}_{2} \mathrm{O}\right)_{4}$} & 117 \\
{$\left[\mathrm{Ag}_{2}(\text { bet })_{2}\left(\mathrm{Tf}_{2} \mathrm{~N}\right)\right]_{\left[\mathrm{Tf}_{2} \mathrm{~N}\right]}$} & 127 \\
{$\left[\mathrm{Hg}(\text { bet })_{2}\right]\left[\mathrm{Tf}_{2} \mathrm{~N}\right]_{2}$} & 111 \\
{$\left[\mathrm{Y}_{2}(\text { bet })_{8}\right]\left[\mathrm{Tf}_{2} \mathrm{~N}\right]_{6} \cdot 2 \mathrm{H}_{2} \mathrm{O}$} & 109 \\
{$\left[\mathrm{La}_{2}(\text { bet })_{8}\right]\left[\mathrm{Tf}_{2} \mathrm{~N}\right]_{6} \cdot 4 \mathrm{H}_{2} \mathrm{O}$} & 78 \\
{$\left[\mathrm{Pr}_{2}(\text { bet })_{8}\right]\left[\mathrm{Tf}_{2} \mathrm{~N}\right]_{6} \cdot 4 \mathrm{H}_{2} \mathrm{O}$} & 86 \\
{$\left[\mathrm{Nd}_{2}(\text { bet })_{8}\right]\left[\mathrm{Tf}_{2} \mathrm{~N}\right]_{6} \cdot 4 \mathrm{H}_{2} \mathrm{O}$} & 90 \\
{$\left[\mathrm{Sm}_{2}(\text { bet })_{8}\right]\left[\mathrm{Tf}_{2} \mathrm{~N}\right]_{6} \cdot 2 \mathrm{H}_{2} \mathrm{O}$} & 88 \\
{$\left[\mathrm{Eu}_{2}(\text { bet })_{8}\right]\left[\mathrm{Tf}_{2} \mathrm{~N}\right]_{6} \cdot 2 \mathrm{H}_{2} \mathrm{O}$} & 95 \\
{$\left[\mathrm{Gd}_{2}(\text { bet })_{8}\right]\left[\mathrm{Tf}_{2} \mathrm{~N}\right]_{6} \cdot 2 \mathrm{H}_{2} \mathrm{O}$} & 94 \\
{$\left[\mathrm{~Tb}_{2}(\text { bet })_{8}\right]\left[\mathrm{Tf}_{2} \mathrm{~N}\right]_{6} \cdot 2 \mathrm{H}_{2} \mathrm{O}$} & 88 \\
{$\left[\mathrm{Dy}_{2}(\text { bet })_{8}\right]\left[\mathrm{Tf}_{2} \mathrm{~N}\right]_{6} \cdot 2 \mathrm{H}_{2} \mathrm{O}$} & 88 \\
{$\left[\mathrm{Ho}_{2}(\text { bet })_{8}\right]\left[\mathrm{Tf}_{2} \mathrm{~N}\right]_{6} \cdot 2 \mathrm{H}_{2} \mathrm{O}$} & 88 \\
{$\left[\mathrm{Er}_{2}(\text { bet })_{8}\right]\left[\mathrm{Tf}_{2} \mathrm{~N}\right]_{6} \cdot 2 \mathrm{H}_{2} \mathrm{O}$} & 94 \\
{$\left[\mathrm{Tm}_{2}(\text { bet })_{8}\right]\left[\mathrm{Tf}_{2} \mathrm{~N}\right]_{6} \cdot 2 \mathrm{H}_{2} \mathrm{O}$} & 97 \\
{$\left[\mathrm{Yb}_{2}(\text { bet })_{8}\right]\left[\mathrm{Tf}_{2} \mathrm{~N}\right]_{6} \cdot 2 \mathrm{H}_{2} \mathrm{O}$} & 89 \\
{$\left[\mathrm{Lu}_{2}(\text { bet })_{8}\right]\left[\mathrm{Tf}_{2} \mathrm{~N}\right]_{6} \cdot 2 \mathrm{H}_{2} \mathrm{O}$} &
\end{tabular}


Figure S5. Experimental setup for pyroelectric measurements
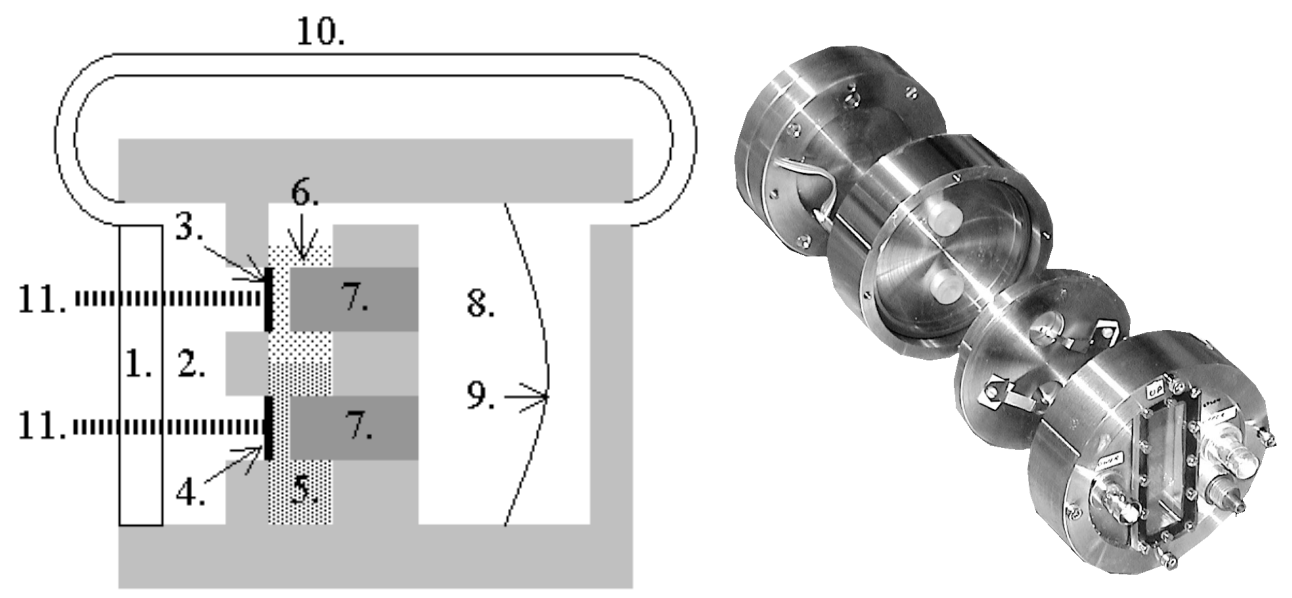

1. window transmitting light from LEDs to sensors

2. air gap

3. upper pyroelectric $\mathrm{LiTaO}_{3}$ sensor

4. lower pyroelectric $\mathrm{LiTaO}_{3}$ sensor

5. sample phase with highest density

6. sample phase with lowest density

7. gold coated copper backing cylinders

8. air and vapour compartment

9. flexible Teflon ${ }^{\circledR}$ membrane for pressure equalization

10. connection tube for pressure equalization

11. light beams from LEDs 